\title{
A Fast Estimation Method for Satellite in Orbit Management
}

\author{
Zhen Cui ${ }^{1,2, a}$, Ye Ji ${ }^{1,2}$, Bin Chen ${ }^{1}$ and Yang Yang ${ }^{1}$ \\ ${ }^{1}$ Beijing Institude of Control Engineering, Beijing, 100094, China \\ ${ }^{2}$ Science and Technology on Space Intelligent Control Laboratory, Beijing,100094, China
}

\begin{abstract}
The management of satellites in orbit requires accurate and rapid processing to minimize the impact of failures. Especially for fault handling involving energy security, the real-time nature of the process plays a decisive role. This paper proposes a fast estimation method based on satellite telemetry data. Through the analysis of the shape of the telemetry curve, the geometric method, the correlation analysis of the telemetry data on the satellite, or the physical principles, the required parameters are quickly obtained to facilitate rapid emergency processing. This method is applied to satellite in-orbit management, which can greatly improve the real-time performance of fault processing, and has good engineering practical value.
\end{abstract}

\section{Introduction}

There are at least two differences between satellite inorbit management and satellite manufacturing, that is, inorbit operations have higher and more stringent requirements for correctness and real-time performance. Once the satellite's in-orbit operation is wrong or the best processing window is missed, the impact is often greater and the consequences are more serious. For example, in the ground test or simulation of a satellite, if an operation error causes the satellite to switch from normal mode to sun capture mode, the device will usually be restarted to continue testing, but if similar problems occur in orbit operation, satellite service will be interrupted. The consequences and impacts are serious. For another example, in ground testing or simulation, if the attitude diverges or the rotation angle of the solar array is wrong, it usually does not cause energy security problems. However, for satellites in orbit, especially those launched in the early days, satellites with weak autonomous functions, if errors cannot be quickly processed, or the best processing window is missed, it may cause serious consequences affecting satellite energy security.

In addition, the following methods are usually adopted for emergency treatment of in-orbit faults. First analyze the phenomenon, then program the simulation to obtain the required parameters, and then send them to the satellite for troubleshooting. This method has two problems in engineering practice. One is insufficient realtime performance, which makes it difficult to perform programming and simulation calculations at the fault processing site, and the other is that calculation errors sometimes occur. This leads to the so-called "accurate but

${ }^{a}$ Corresponding author: $5277981 @ q q . c o m$ incorrect" calculation result. Although the calculation result is accurate to a few digits after the decimal point, it is an invalid result, and the consequences of this situation are often very serious.

Some scholars have researched how to avoid the problem of "accurate but incorrect" during simulation and calculation. For example, Lu Jiaxi proposed a method of estimation in research ${ }^{[1]}$. By establishing a simplified model, a reasonable estimate of the calculation results can be obtained, which can effectively avoid "precise errors" in analytical calculations. Yang Weilian compares "simulation" and "approximate" [2], and illustrates the significance and importance of "approximate" in engineering through some examples. Yang Weilian mentioned: "Simple models are easier to understand and easier to check than complex models, so it is easier to ensure their correctness. Conversely, the more complex the model, the more likely it is to make mistakes in the application process."

This paper proposes a fast estimation method based on satellite telemetry parameters, which is an engineering approximate calculation method. This method can improve the accuracy and real-time performance of inorbit satellite management, and has certain engineering value. This method can effectively avoid "precise errors" in analytical calculations by establishing a simplified model and quickly estimating the reasonable range of the parameters. This is exactly what Keynes calls "Better be roughly right than precisely wrong".

\section{Fast estimation method}

\subsection{Usage of estimation methods}


In satellite in-orbit management, the application of the fast estimation method is roughly derived from the following requirements.

1) Accurate calculations often require complex formulas or programming, and they are often not equipped for satellite emergency processing.

The occurrence of satellite failures is often unpredictable and may occur at any time. This requires emergency treatment in the field, and it is difficult to have conditions such as programming simulation at this time. The estimation method can be quickly processed according to the relevant telemetry parameters on the satellite.

2) Satellite emergency processing usually requires rapid and effective processing methods, and does not necessarily require accurate results.

For example, when the satellite attitude deviates from the target attitude in orbit, and the solar array deviates from the target rotation angle, which affects satellite service or energy security, the attitude or solar array rotation angle needs to be adjusted quickly to ensure energy. At this time, the estimation method based on satellite telemetry parameters is often faster.

3) The estimation method often helps to quickly determine the cause of the fault.

When a satellite fails, comprehensive analysis is required to determine the cause of the failure. For example, when the attitude angle fluctuates, it is necessary to quickly analyze the attitude angle, the output of each sensor, the rotation angle of the solar array, the rotation speed of the reaction wheel, angular momentum, and the orientation of the sun and the moon. The estimation method helps to quickly analyze the parameters and quickly determine the cause of the failure.

4) The estimation method and the programming simulation method are compared with each other, which is helpful to improve the accuracy of in-orbit processing.

The estimation method is simple and fast, and the physical or geometric meaning is clear. The rationality of the simulation results can be judged to prevent the simulation calculation from appearing "accurate but incorrect" results. The simulation calculation can accurately estimate the error of the estimation method and prevent large errors in the estimation method.

\subsection{Fast estimation based on curve topography}

In-orbit management and troubleshooting generally need to be clear about the question of "where is the sun", that is, the elevation and azimuth information of the sun vector. If the programming simulation method is adopted, the representation of the sun direction vector in the orbit coordinate system needs to be calculated.

$$
\mathbf{S}_{\mathrm{o}}=\mathbf{A}_{\text {ou }} \mathbf{A}_{\text {oi }} \mathbf{S}_{\mathrm{i}}
$$

Where $\mathbf{S}_{\mathrm{i}}$ is the representation of the sun vector in the inertial coordinate system. It is defined as:

$$
\mathbf{S}_{\mathrm{i}}=\left[\begin{array}{lll}
S_{i x} & S_{i y} & S_{i z}
\end{array}\right]^{\mathrm{T}}
$$

$\mathbf{S}_{\mathrm{i}}$ needs to be calculated according to the ephemeris of the sun.

$\mathbf{A}_{\text {ou }}$ and $\mathbf{A}_{\text {oi }}$ are $3 \times 3$ transformation matrices related to the satellite orbit, $\mathbf{A}_{\text {ou }}$ is defined as:

$$
\mathbf{A}_{\text {ou }}=\left[\begin{array}{ccc}
\cos u & 0 & \sin u \\
0 & 1 & 0 \\
-\sin u & 0 & \cos u
\end{array}\right]
$$

$\mathbf{A}_{\mathrm{oi}}$ is defined as:

$$
\mathbf{A}_{\mathrm{oi}}=\left[\begin{array}{ccc}
\cos i \cos \Omega & -\sin i & \cos i \sin \Omega \\
\sin i \cos \Omega & \cos i & \sin i \sin \Omega \\
-\sin \Omega & 0 & \cos \Omega
\end{array} \mid\right.
$$

Where $u=\omega+f, \omega$ is the argument of periapsis, $f$ is the true anomaly, $i$ is the orbit inclination and $\Omega$ is the right ascension of ascending node ${ }^{[3]}$.

$\boldsymbol{S}_{o}$ is the representation of the sun direction vector in the orbit coordinate system. The $\boldsymbol{S}_{o}$ can be calculated before the subsequent calculation of various angles. This process is relatively complicated, and it is difficult to judge the rationality of the results. Moreover, it is difficult to have the conditions to perform such calculations at the site of the fault treatment.

The following describes a method for quickly determining the solar position based on the shape of the satellite telemetry curve. According to this method, the solar position of the satellite at each time in a future orbit cycle can be quickly estimated and predicted.

For example, for IGSO navigation satellites ${ }^{[4-6]}$, the continuous maneuver control of the yaw angle keeps the sun vector in the $X_{\mathrm{b}} O_{\mathrm{b}} Z_{\mathrm{b}}$ plane of the satellite body coordinate system, and controls the rotation angle of the solar array so that the normal of the solar array always points in the direction of the solar vector [7-11]. The position of the sun includes the sun elevation angle $\theta_{\mathrm{s}}$ and the sun azimuth angle $\alpha$. The sun azimuth angle $\alpha$ is the angle between the projection of the sun vector on the orbital $X_{\mathrm{o}} O_{\mathrm{o}} Z_{\mathrm{o}}$ plane and the $+Z_{\mathrm{o}}$ axis, and it is positive from the $+Z_{\mathrm{O}}$ axis $+X_{\mathrm{O}}$ axis rotation. The elevation angle $\alpha$ of the sun is the angle between the sun vector and the orbital plane, with the sun vector pointing to the orbital system $+Y_{\mathrm{o}}$ plane as positive.

The yaw angle curve of an orbital period contains the information of the solar elevation angle $\theta_{\mathrm{s}}$, as shown in Figure 1.

$$
\theta_{\mathrm{S}}=\min (\psi(\mathrm{t}))
$$


Where $\psi(t)$ is the yaw angle of the satellite.

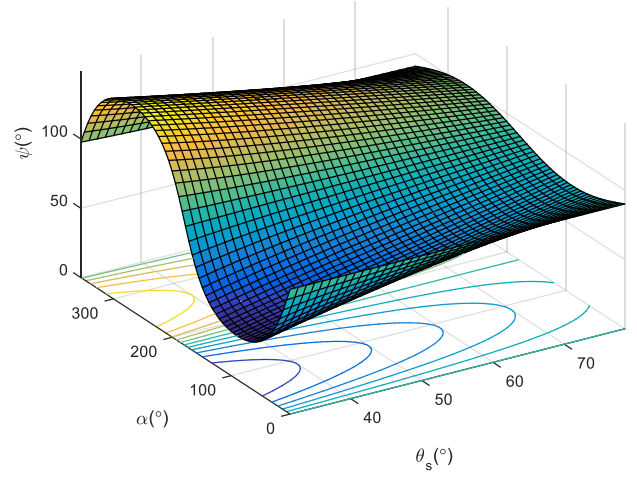

Figure 1. Variation of yaw angle with $\theta_{\mathrm{s}}$ and $\alpha$

The solar array angle curve of one orbital period contains the information of the solar azimuth angle.

$$
\cos \alpha \cos \theta_{\mathrm{S}}=\cos \left(\beta_{\mathrm{sada}^{-}}{ }^{-\pi}\right)
$$

$\beta_{\text {sada }}$ is the angle of the solar array.

For example, for a GEO navigation satellite, since the control targets of the three-axis attitude are all $0^{\circ}$, the solar position can be estimated based on the output of each solar sensor or the rotation angle of the solar array.

The output angle curve of the solar sensors DSS13 and DSS43 in one orbit period contains the information of the solar elevation angle $\theta_{\mathrm{S}}$

$$
\left|\theta_{\mathrm{S}}\right|=\min \left(\alpha_{\mathrm{DSS}}(\mathrm{t})\right)
$$

Among them, $\alpha_{\text {DSS }}(\mathrm{t})$ is the output angle information of digital solar sensors DSS13 and DSS43.

The solar array angle curve of one orbital period contains the information of the solar azimuth angle $\alpha$.

$$
\alpha=\beta_{\text {sada }^{-}}-\pi
$$

\subsection{Fast estimation based on geometric relations}

The following uses an example in engineering practice to illustrate. In the in-orbit management of the MEO navigation satellite, the satellite is in a yaw dynamic offset mode. The continuous maneuver control of the yaw angle keeps the sun vector in the $X_{\mathrm{o}} O_{\mathrm{o}} Z_{\mathrm{o}}$ plane of the satellite body coordinate system, and controls the rotation angle of the solar array so that its normal line always faces the direction of the sun vector. Assume that due to the need for phase adjustment, or the need for emergency treatment of the fault, the satellite needs to enter the phase adjustment mode and make the yaw angle $0^{\circ}$. At this time, how to adjust the rotation angle of the solar array so that it faces the sun as much as possible?

If the programming simulation method is adopted, the expression of the solar vector in the orbit coordinate system still needs to be calculated according to formula
(1). In fact, the calculation of the space angle has nothing to do with the choice of the coordinate system. If the method of coordinate system and transformation matrix is not adopted, the telemetry parameters of the satellite can be obtained, and the simple and fast estimation can be performed through the geometric relationship.

It can be known from formula (5) that the solar elevation angle $\theta_{\mathrm{S}}$ can be obtained from the yaw curve of the satellite in an orbital period, and the solar array rotation angle $\beta_{\text {sada }}$ is a direct telemetry parameter of the satellite. Then through simple geometric calculations, the optimal rotation angle of the solar array can be obtained.

$$
\cos \beta_{0} \cos \theta_{\mathrm{S}}=\cos \left(\beta_{\mathrm{sada}^{-}}{ }^{-\pi}\right)
$$

Then the satellite yaw angle needs to be adjusted as follows.

$$
\Delta \beta_{\text {sada }}=\beta_{\text {sada }}-\left(\pi+\beta_{0}\right)
$$

Figure 2 shows the surface of $\Delta \beta_{\text {sada }}$ as it changes with $\theta_{\mathrm{S}}$ and $\beta_{\text {sada }} \cdot \Delta \beta_{\text {sada }}$ is monotonically decreasing or rising between $\theta_{\mathrm{S}}$ and 0 . When $\theta_{\mathrm{S}}$ is large, the shape of the surface is equivalent "flat". In engineering practice, for example, in the case of emergency handling of a fault, or when it is related to energy security, if the simulation calculation conditions are not available at the fault processing site, $\Delta \beta_{\text {sada }}$ can estimate according to a linear method to adjust the rotation angle of the solar array to ensure satellite energy.

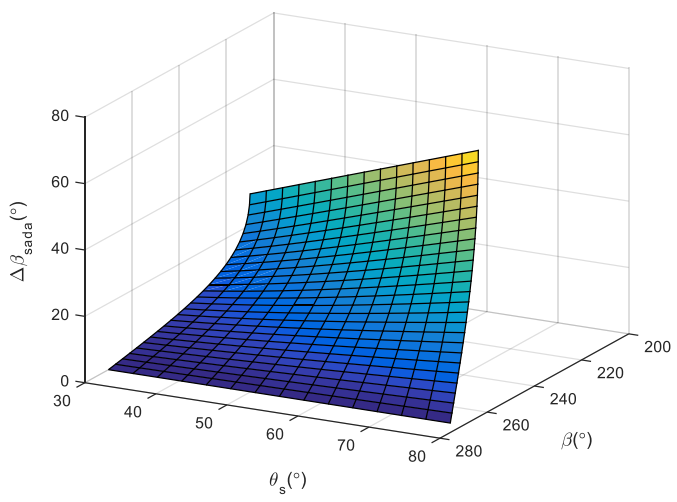

Figure 2. $\Delta \beta_{\text {sada }}$ changes with $\theta_{\mathrm{S}}$ and $\beta_{\text {sada }}$

\subsection{Fast estimation based on correlation of telemetry data}

In the process of satellite in-orbit management and inorbit fault processing, since we can directly obtain a large amount of telemetry data at the processing site, we should make full use of the correlation between these telemetry parameters for rapid estimation. 
For example, an IGSO navigation satellite has entered solar capture mode due to incorrect orbit data. Because the fault occurs at night and there may be satellite eclipse, it is necessary to immediately inject the parameters related to satellite eclipse. However, since the failure occurred at night, it was difficult to carry out the simulation calculation immediately. At this time, a quick estimation can be performed based on the yaw angle of the previous orbit period and the rotation angle parameters of the solar array.

According to formula (5), determine whether the satellite is in the period of eclipse or not based on the curve shape of the yaw angle of the previous orbital period.

$$
\theta_{\mathrm{S}}=\min (\psi(\mathrm{t}))>R_{e}+R_{\mathrm{S}}
$$

If formula (11) is satisfied, it is not in the period of eclipse, otherwise it is in the period of eclipse. Where $R_{e}$ is the half cone angle of the earth and $R_{S}$ is the half cone angle of the sun. If the satellite is in the eclipse period, the parameters related to the eclipse can be obtained by querying the solar array rotation telemetry data in the normal mode of the previous orbital period.

\subsection{Fast estimation based on physical principles}

Taking the failure of the momentum wheel on the satellite as an example, according to the principle of conservation of angular momentum, the shape of the speed curve of each momentum wheel after the failure and how to recover can be estimated. Neither simulation nor calculation is required, nor is it necessary to switch to earth pointing mode or position maintaining mode for processing. For example, a GEO navigation satellite is equipped with four pyramid-shaped momentum wheels to form a wheel control system ${ }^{[12]}$. If the entire satellite has zero momentum and a certain momentum wheel is powered off, since jet unloading is not allowed, the satellite keeps angular momentum conserved, so the speed of the other three momentum wheels will also decrease simultaneously. If the momentum wheel is restarted, and then the restarted momentum wheel is restored to the target speed, other momentum wheels will naturally be restored to the target speed simultaneously.

\subsection{Comprehensive of fast estimation method}

In engineering practice, especially for the emergency treatment of in-orbit faults, it is necessary to comprehensively apply the above-mentioned multiple methods for rapid emergency treatment. The comprehensive application of the rapid estimation method requires attention to the following:

1) When emergency handling of satellite in-orbit faults, the parameters are estimated based on the morphological characteristics of the curve, and the morphology of the curve in the recent orbital cycles before the failure should be identified. It is necessary to identify which is the correct data and which is the data at the time of failure. Try to avoid using only one orbit period of telemetry data for estimation, and prevent the use of wrong telemetry data.

2) In the case of non-emergency in-orbit processing, when the conditions for simulation calculation are available, simulation calculation based on ephemeris and rapid estimation based on telemetry parameters should be performed at the same time. Two different calculation methods are mutually checked and verified to avoid calculation errors. Fast estimation can avoid "accurate errors" in simulation calculations, and simulation calculations can prevent large estimation errors from occurring in rapid calculations.

3) When a fast estimation method based on the correlation of telemetry parameters is used, there are often multiple estimation methods for the same parameter, and multiple methods should be verified with each other to prevent estimation errors. For example, satellite attitude information, measurement information from multiple sensors, solar array rotation angles and other satellite telemetry data are all related to solar position information. Comprehensive verification should be performed through multiple methods and multiple associated telemetry data.

4) If the satellite attitude diverges, fast attitude recovery is needed, or the solar array angle needs to be adjusted quickly if the satellite energy security is endangered. When the simulation calculation conditions are not available, a fast estimation method based on telemetry parameters should be selected for processing. If the failure is not handled in a timely manner, it will lead to serious risks affecting satellite services or safety.

\section{Conclusion}

Aiming at the long-term in-orbit management of satellites and emergency handling of in-orbit faults, this paper proposes a fast estimation method based on on-board telemetry parameters and their correlation analysis, and illustrates the application value of this method through specific examples. Compared with the traditional programming or simulation method, this method can be quickly processed at the fault site, the calculation is simple, the geometric significance is obvious, and it can be applied to the practice of satellite in-orbit management.

\section{References}

1. Lu Jiaxi. On the "rough estimation" method in scientific research. Academician Thinking Volume III, [M],Hefei: Anhui Education Press, 2001: 130136.

2. Yang Weilian. Simulation and approximationdiscussion on engineering methodology. Proceedings of the 2004 Academic Symposium of the Aircraft General Professional Committee of China Aerospace Society, 2005, 133-136. 
3. Zhang Renwei. Satellite Orbit Attitude Dynamics and Control [M]. Beijing: Beijing University of Aeronautics and Astronautics Press, 1998:6-8.

4. Mao Yue, Song Xiaoyong, Wang Wei, et al. Beidou IGSO and MEO navigation satellites' yaw-steering and orbit-normal attitude control modes and solar radiation pressure difference analysis $[\mathrm{J}]$. Science of Surveying and Mapping, 2015, 40 (8) : 129134.

5. Mao Yue, Song Xiaoyong, Wang Wei, et al. IGSO satellite orbit determining strategy analysis with the yaw-steering and orbit-normal attitude control mode switching[J]. Geomatics and Information Science of Wuhan University, 2014, 39 (11) : 1352-1356.

6. Li Xiao Jie, Liu Xiao Ping, Xin Jie, et al. Attitude Control Modes and Characteristic Analysis for BeiDou Satellites[J]. Journal of Geomatics, 2019, 44 (5) : 69-72.

7. Mao Yue, Song Xiao Yongoing, Jia Xiao Lin. Analysis about Parameters Selection Strategy of ECOM Solar Radiation Pressure Model for BeiDou Satellites[J]. Acta Geodaetica et Cartographica Sinica, 2017, 46 (11) : 1812-1821.
8. Liu Yu Xi, Jia Xiao Lin, Ruan Ren Gui. Beidou IGSO Satellite Orbit Determination Precision Analysis Based on New Attitude Control Mode[J]. Journal of Geodesy and Geodynamics, 2017, 37 (6) : 614-617.

9. Ji Guo Febng, Yang Zhi Qiang, Jia Xiao Lin, et al. A Study on the Impact of Yaw-Attitude and Solar Radiation Model on Precise Orbit Determination for Beidou Navigation Satellites[J]. Journal of Geodesy and Geodynamics, 2018，38 (4) : 374-380.

10. Wang Chen, Guo Jing, Zhao Qi Le. Impact of Yaw Attitude on GPS/GLONASS Orbit and Clock Solutions $[\mathrm{J}]$. Geomatics and Information Science of Wuhan University，2017，42（5）: 624-629.

11. Zhou Pei Yuan, Du Lan, Fang Shan Chuan, et al Analysis of Characteristics of QZSS Satellite Orbit and Clock Products during Yaw Attitude Model Switching[J]. Acta Geodaetica et Cartographica Sinica, 2016, 45 (3) : 274-281.

12. Lei Yongjun, Yuan Li. Angular momentum envelope analysis and angular momentum management of spacecraft[J]. China Space Science and Technology, 2017, 37 (6) : 1-9. 